\title{
ROBOTIC SYSTEM FOR ROTATION, RECOGNITION, DIGITALIZATION \& DATA IDENTIFICATION FROM SCIENTIFIC CARD-FILES IN OLD FILING-CABINETS
}

\author{
Верховцев А.Г. ${ }^{1}$, Градов О.В., Нотченко А.В., ЛНСМ НЦН РАМН, 2012
}

ВВЕДЕНИЕ

Организация информации в библиотеках и архивах требует компьютеризации и переноса информации картотек в машинно-читаемый вид $[1,2]$. Тенденция на переход библиотек от простого хранения и выдачи книг к созданию автоматизированных медиа-центров на их основе [3-5] делает эту задачу весьма актуальной. Проектирование новых современных библиотек и перенос в них содержимого старых библиотек [6-8] также требует учета, становящегося весьма длительным и ресурсозатратным при ипользовании ручного труда библиотекарей. В особенности, это касается научных и академических библиотек [9], где разнообразие форматов хранящегося продукта и необходимость его указания в разных системах цитирования $[10,11]$ делает необходимым многократный ввод наименований отпечатков статей, препринтов, протокольной литературы и т.д. Необходимость использования метаданных [12] при библиотечном индексировании [13-16], а также создании он-лайн каталогов литературы [1719], делает неизбежной разработку технических средств для оцифровки картотек и каталогов библиотек [20], в особенности необходимой для организации сетей научно-технических библиотек [21] и удаленной работы с документами (в том числе - в распределенных группах) при электронном документообороте в архивах $[22,23]$ научно-исследовательских центров.

\footnotetext{
${ }^{1}$ Псевдоним специалиста, работающего в организации, не допускающей разглашения информации. Ранее статья выходила с именем данного специалиста в издании с усеченными выходными данными по причине сбоя в системе авторской регистрации. 
Следует отметить, что аналогичная задача стоит не только перед операторами библиотечных картотек, но и перед владельцами и архивными работниками в архивах фотокарточек, карт и изображений $[24,25]$, за тем исключением, что в этом случае должно применяться не только распознавание текста карт, но и машинное распознавание изображений [26].

Существование интеллектуальных основ восприятия информации человеком делает неизбежной применение этих основ при организации информации в библиотеках [27] и, как следствие, социального восприятия информации в них [28]. От этого зависит эффективность менеджмента, маркетинга и учета (как коррелята количественной эффективности чтения) в библиотеках [29-32]. Идеальным решением был бы случай "менеджмента без менеджеров" [33] в библиотеках, когда сами читатели определяют и онлайнформируют реляции списков литературы, исходя из чего формируется планы [34]. В старых библиотеках этот процесс осложнен работой с картотеками. Известно, однако, что читатель имеет меньшую скорость чтения карточек, чем автоматика. В связи с этим, имеется необходимость в создании аппарата для быстрого считывания карточек, снятия информации с них и распознавания кейвордов, введенных пользователем. Это могло бы упростить и исследование фондов библиотекарями [35], в особенности - той литературы и тех картотек, которые были оформлены задолго до появления рекомендаций и стандартов ВТО и специализированных библиометрических технологических стандартов [36, 37]. Онлайн-поиск [38] можно совместить с роботизированным поиском в картотеках, что особенно удобно для маленьких библиотек с небольшими картотеками [39]. Аналогичное может быть применено в картотеках музеев [40] и в архивном деле [41-44], при взаимно-однозначном сопоставлении лейблов единиц хранения карточкам с их описанием [45].

Динамичность подобной библиотечной организации [46] позволяет не полностью переводить архивы в базы данных, загружая распознаваемые карточки в память по мере востребования их пользователем, отделяя тем 
самым релевантную информацию от нерелевантной. Вместе с тем, можно создавать глобальные базы данных, используя читателей, пользующихся машиной считывания карточек, в "краудсорсинговом" труде при выполнении ими своих поисковых запросов. Тем самым преодолеваются возможные вероятные противоречия между требованиями международных организаций коммерческого плана [47] и системой открытого доступа (Open Projects) в рамках библиотечной этики [48] без грантов и необходимого для их получения фандрайзинга [49] в штатном режиме работы библиотеки. С другой стороны, это de facto участвует в преодолении силами читателей противоречия между необходимостью серьёзного академического поиска и его скоростью [50]. Это, в целом, не противоречит классическим принципам организации библиотечных изысканий [51], а творчески дополняет их в соответствии с социальными и техническими возможностями современного периода, также как когда-то введение самих картотек, печатей экслибрисной маркировки и т.п. было новым для книжного дела [52].

\section{КОНСТРУКЦИЯ УСТАНОВКИ.}

Система состоит из механизированной части на основе шаговых двигателей с числовым программным управлением, ротатора (аналогичного стоящему на счетчиках банкнот) $)^{2}$, камеры со светозащитным тубусом, по размерам совпадающим с размером ящика картотеки, и индикатора, изображение которого вместе с изображением карты выводится на специализированную карту компьютера.

Нами была использована камера "SONY", подключенная через блок "SONY" к плате видеозахвата ЭВМ. Она насаживалась на тубус, снятый с пластиночной фотокамеры для микроскопов образца 1950-х гг., внутри и снизу которого монтировалась ротационная часть установки. Общий вид

\footnotetext{
2 в различных проектах по оцифровке книг, где необходимо перелистывание страниц (что эквивалентно перелистыванию карточек в картотеке) используются как автоматические, так и неавтоматические способы ротации: http://diybookscanner.org/, http://www.youtube.com/user/bookscanner, http://www.geocities.jp/takascience/lego/fabs en.html
} 
данной конструкции при горизонтальном и вертикальном типе съёмки, соответствующем расположению карточек в картотеке, показан на илл. 1 a, б.

В нижней части микрофототубуса монтировался индикатор, электроника управления которым показана на илл. 2 а. Индикатор управлялся перепрошитым контроллером телефонии с автоматическим определителем номера. В итоге в кадр, помимо изображения карточки, попадало изображение индикатора, показанное на илл. 2 б. Затем автоматически на ПК строилась изолинейная визуализация его изображения и по высшим изолиниям или с использованием операции эрозии получалось четкое изображение, паттерн которого распознавался полностью автоматически с использованием специализированных программ. Вид низко-дискретного изолинейного картирования индикации приведен на илл. 3.

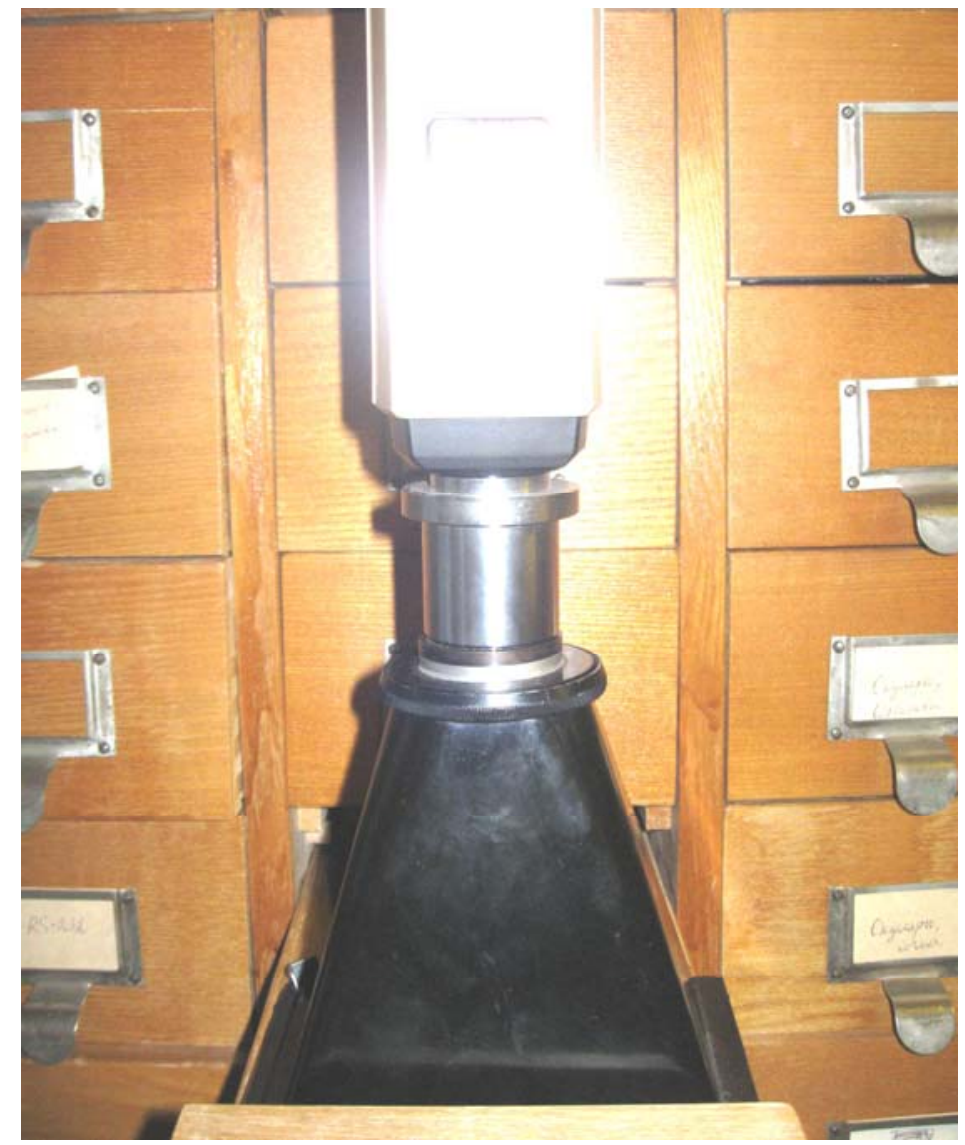

a

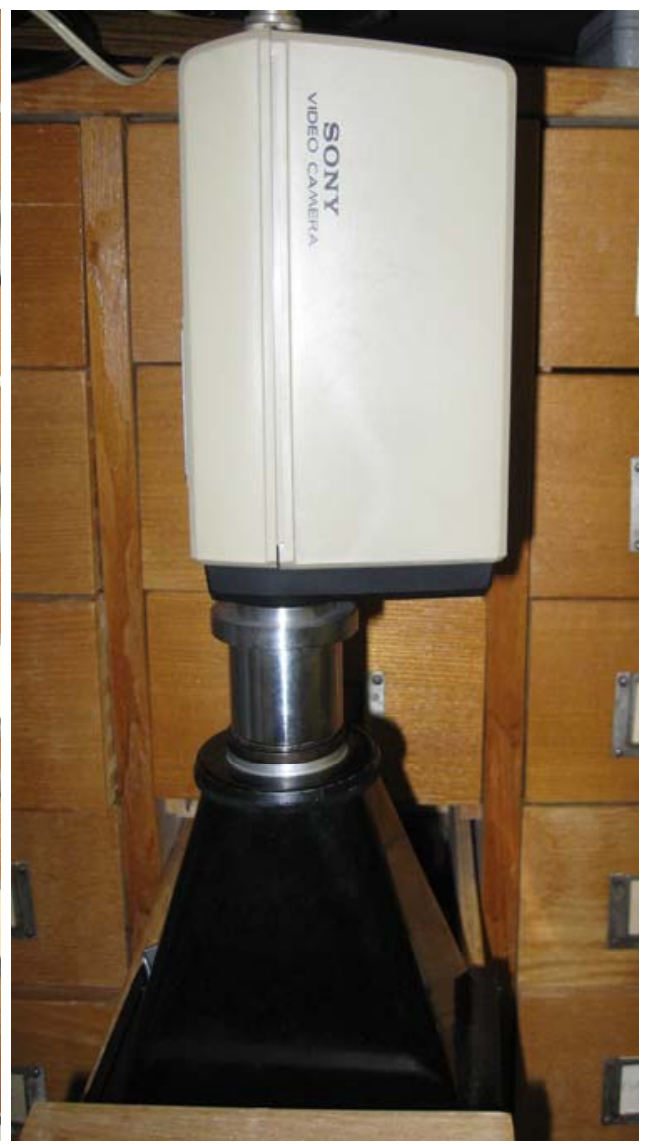

б

Илл. 1: Общий вид данной конструкции при горизонтальном (а) и вертикальном (б) типе съёмки 
После размещения установки на движущейся платформе на ящике и распознавания карточек в ящике датчиком объёма или фотоэлементом, шаговые двигатели, управляемые от ПК через плату, начинают приводить в движение лопасти, листающие карточки (система ротатора и управления им является в настоящее время узким местом проекта). Каждое перелистывание сопровождается остановом машины и считыванием информации с карточки. Bсе это с помощью утилиты выдается в компьютерном виде и может заноситься в базы данных и систематизироваться. Система умеет обращаться при помощи оператора к сети для уточнения названия по маске.

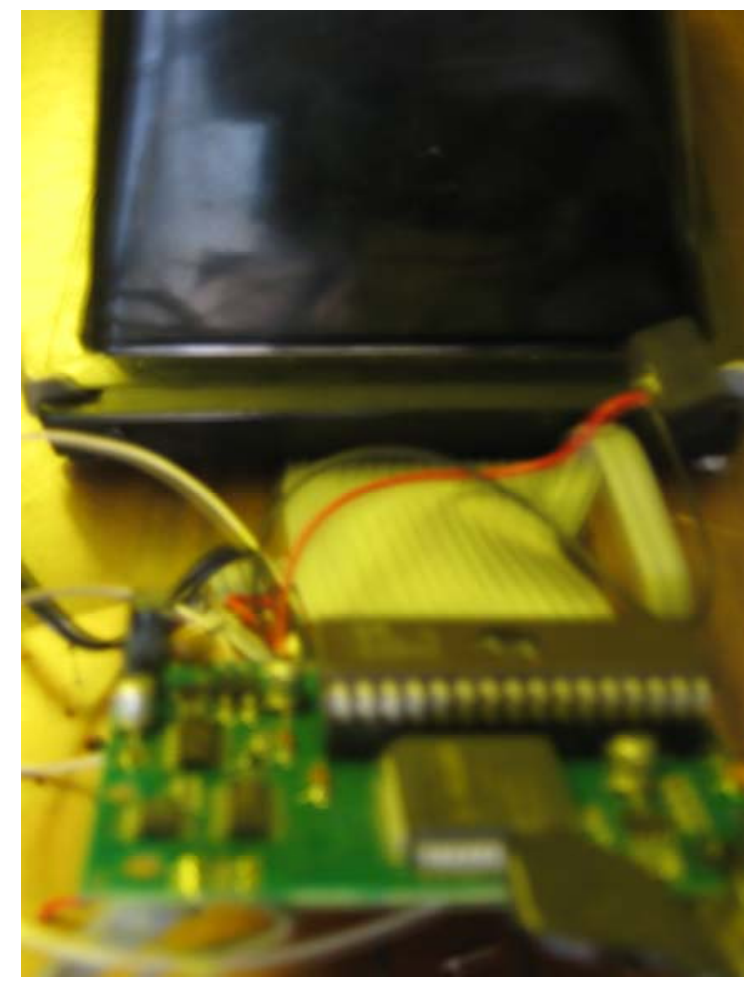

a

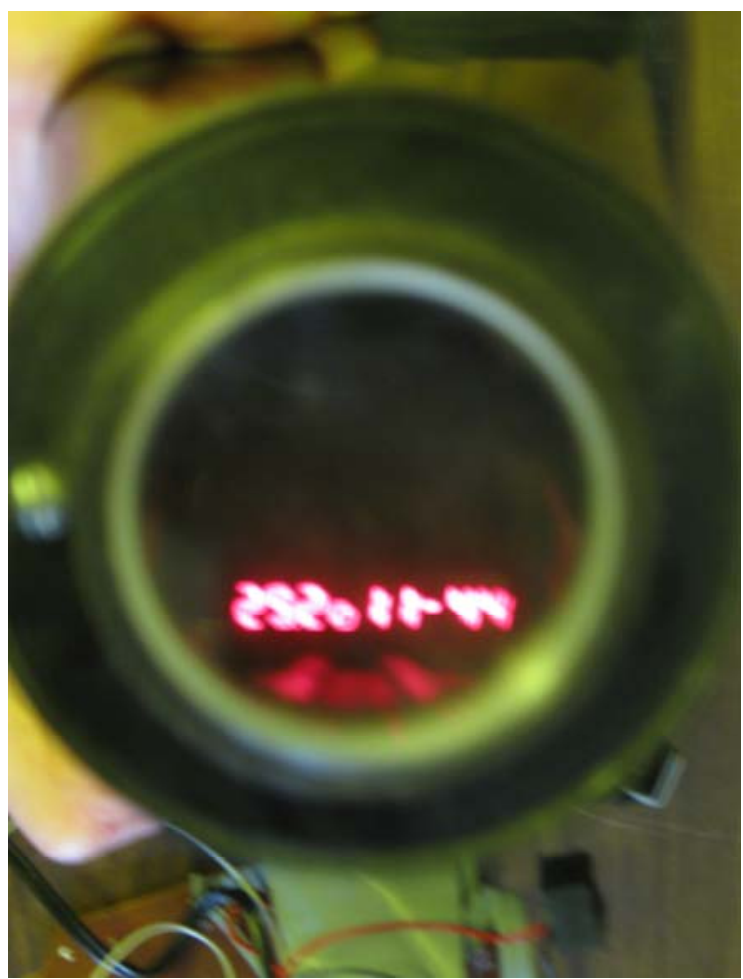

6

Илл. 2: Система индикации: а - электроника, управляющая индикацией; б индикатор в поле кадра

Возможно распознавание также карт с баркодами, но таковые редко встречаются в картотеках библиотек старого типа в России, хотя достаточно распространены в США и ЕС. Для этого система дополняется лазерным источником и системой считывания, замещающей видеоголовку SONY, 
показанную на илл. 1. Опыт работы над такого рода приложениями имеется у авторов, но до сих пор применялся только в методах лазерного баркодинга физиологической информации и лазерных роботизированных установках биофизического назначения [53]. Возможно использовать обратный принцип: дополнение современных электронных картотек привычными для персонала бумажными картотеками с выводом на карточки штрих-кодов современного типа для их машинного распознавания с помощью описанной конструкции, но это не является рациональным при наличии цифровых баз данных.

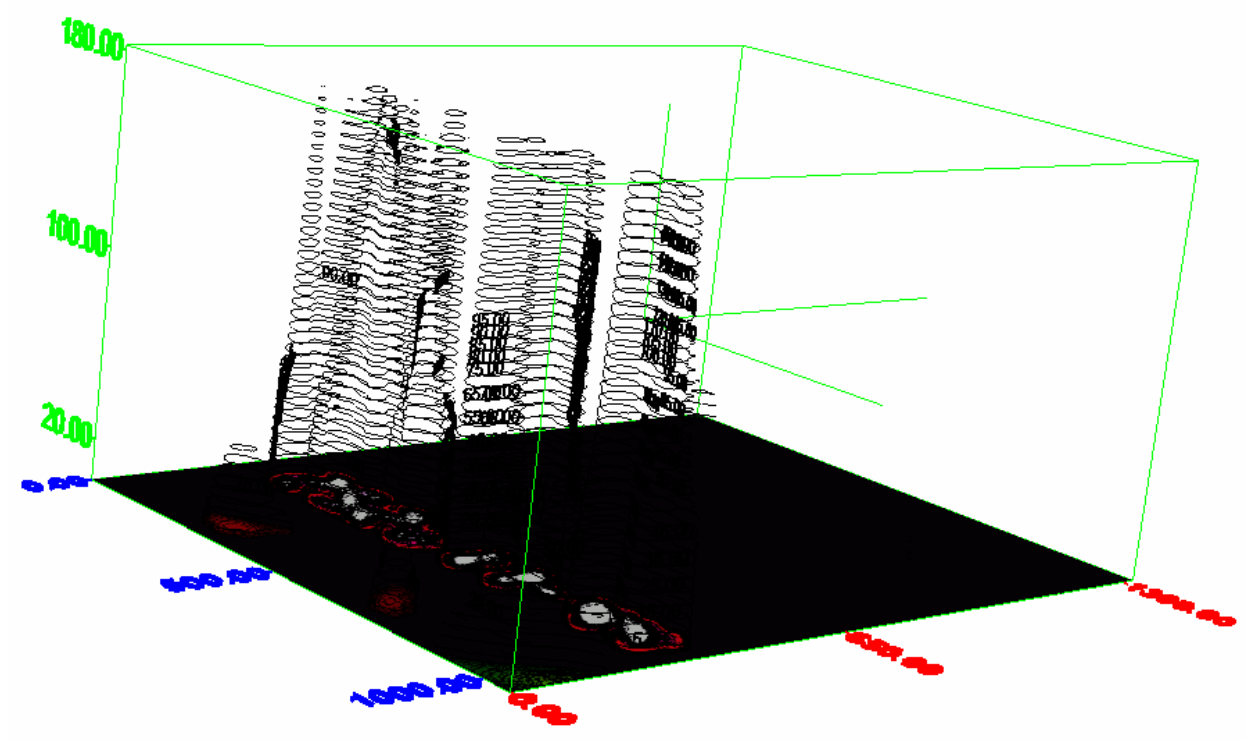

Илл. 3: Изолинейная карта свечения индикатора, путем эрозии приводимая к машинно-дешифруемому состоянию, распознаваемому стандартным ПО.

Различные варианты конструкции данной системы опробованы авторами на спецкартотеке в НЦН РАМН. Распознанные UDC идентифицировались с помощью флэнг-системы на базе объектной модели в стандарте метаданных Dublin Core. В частности для этого косвенно использовалась отечественная UDC-система http://teacode.com/online/udc/about.html, в которой применены следующие объектные свойства Dublin Core: "identifier" - код элемента УДК, "title" - название элемента, "description" - описание элемента УДК, "isPartOf" код надэлемента (суперкласса) данного элемента, "references" - ссылки на элементы УДК, связанные с данным элементом, "isReplacedBy" - информация 
о замене элемента. Путем создания данных гиперссылок облегчалась работа с реляционными базами данных, содержащими идентифицируемый материал.

Для автоматического баркодинга предлагается использовать программы, подобные написанной нами для баркодинга нейрофизиологических данных, но затем адаптированной под нужды библиотечного баркодинга (см. илл. 4). Апробация подобной системы, в частности, проводилась нами на карточках US Patents, имеющих нанесенные на них штрих-коды. Поскольку данное ПО обладает, как можно видеть из приведенного изображения, также и областью угловой привязки данных сканирования кода, ориентация в картотеке при её различных углах поворота и различной ориентации карточек в описываемой роботизированной системе полностью автоматизирована и не требует ввода данных человеком.

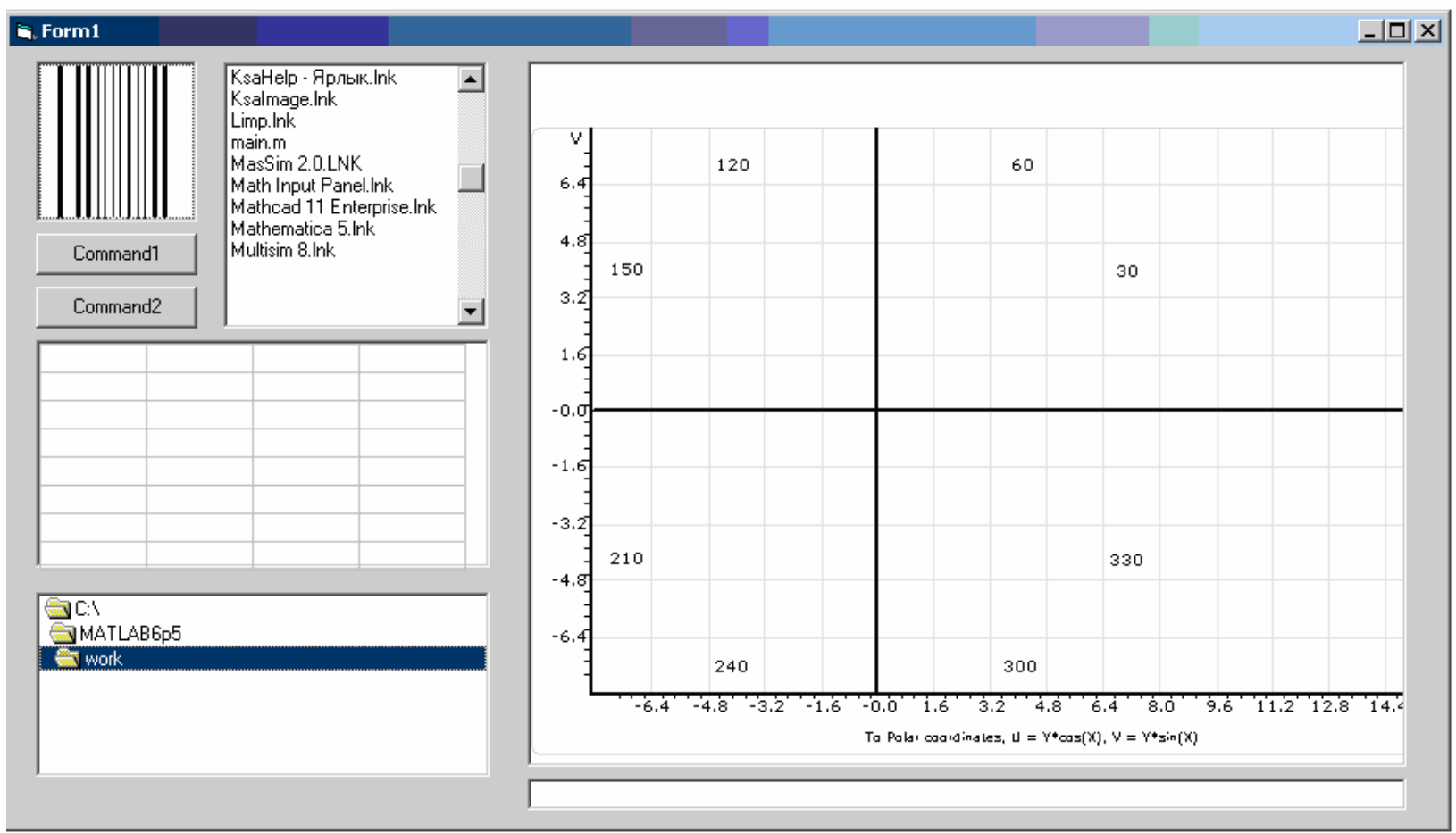

Илл. 4: Программное обеспечение "NeuroBarcoder" [53] после адаптации под распознавание образов сканинга с роботизированной библиотечной системы.

\section{ЗАКЛЮЧЕНИЕ}

Таким образом, описанная система является оптимальным средством для обеспечения дешифруемости информации с бумажных карточек в картотеках 
устаревшего типа в автоматическом режиме. На сканирование одного ящика уходит менее получаса времени даже при низких скоростях передвижения по нему считывающей головки. В связи с этим рекомендуется использование и разработка подобных систем для повседневного применения в библиотеках.

\section{ЛИТЕРАТУРА}

1. Taylor A.G. The Organization of Information (Library and Information Science Text Series), 448 p., Libraries Unlimited, 2003.

2. Taylor A.G., Joudrey D.N. The Organization of Information (Library and Information Science Text Series), 512 p., Libraries Unlimited, 2008.

3. Taylor J. Information Literacy and the School Library Media Center, 168 p., Libraries Unlimited, 2005.

4. Erikson R., Markuson C. Designing a School Library Media Center for the Future, 136 p., Amer. Library Ass. Ed., 2009.

5. Schultz-Jones B. An Automation Primer for School Library Media Centers and Small Libraries, 280 p., Linworth, 2006.

6. Sannwald W.W. Checklist of Library Building Design Considerations, 224 p., Amer. Library Ass. Ed., 2009.

7. Roth M. Masterpieces: Library Architecture + Design, 304 p., Braun Pub., 2010.

8. McCarthy R. Managing Your Library Construction Project, 184 p., Alpha Pub. House, 2011.

9. Stewart S. The Academic Library Building in the Digital Age: A Study of Construction, Planning, and Design of New Library Space, 116 p., Amer. Library Ass., 2010.

10.Pugh M.J. Providing Reference Services for Archives \& Manuscripts (Archival Fundamentals Series), 368 p., Society of Amer. Archivists, 2005)

11.Cassel K.A., Hiremath U. Reference and Information Services in the 21st Century, Second Edition Revised, 450 p., Neal-Schuman Publishers, 2011. 
12.Untner S.S., Lazinger S.S., Weihs J. Metadata and Its Impact on Libraries, (Library and Information Science Text Series), 272 p., Libraries Unlimited, 2005.

13. Mulvany N.C. Indexing Books (Chicago Guides to Writing, Editing, and Publishing), 320 p., University Of Chicago Press, 2005.

14.Indexes: A Chapter from The Chicago Manual of Style, 64 p., University Of Chicago Press, 2010.

15.Fetters L.K. Handbook of Indexing Techniques: A Guide for Beginning Indexers, 109 p., Fetters Infomanagement Co., 2008.

16.Cleveland D.B., Cleveland A.D. Introduction to Indexing and Abstracting, 283 p., Libraries Unlimited, 2000.

17.Haynes E., Fiuntain J.F. Unlocking the Mysteries of Cataloging: A Workbook of Examples, 268 p., Libraries Unlimited, 2005.

18.Chan L.M. Cataloging and Classification: An Introduction, 600 p., Scarecrow Press, 2007.

19. Taylor A.G. Introduction to Cataloging and Classification (Library and Information Science Text Series), 608 p., Libraries Unlimited, 2006.

20.Barth C.D. Convergence of Libraries and Technology Organizations: New Information Support Models, 200 p., Chandos Publishing - Oxford, 2011.

21.Sci Tech Library Networks Within Organizations (Ed. by E. Mount), 162 p., Routledge, 1988.

22.Sutton M.J. Document Management for the Enterprise: Principles, Techniques, and Applications, 400 p., Wiley, 1996.

23. Azad A. Implementing Electronic Document and Record Management Systems, 280 p., Auerbach Pub., 2007.

24.Ritzenthaler M.L., Vogt-O'Connor D., Zinkham H., Carnell B., Peterson K. Photographs: Archival Care And Management, 529 p., Society of Amer. Archivists, 2006.

25.Layedrine B. A Guide to the Preventive Conservation of Photograph Collections, 304 p., Getty Conservation Institute, 2003. 
26. Gascoigne B. How to Identify Prints, Second Edition, 216 p., Thames \& Hudson, 2004.

27. Svenonius E. The Intellectual Foundation of Information Organization (Digital Libraries and Electronic Publishing), 280 p., The MIT Press, 2000.

28. Wildemuth B.M. Applications of Social Research Methods to Questions in Information and Library Science, 421 p., Libraries Unlimited, 2009.

29.Fisher P.H., Pride M.M. Blueprint for Your Library Marketing Plan, 152 p., Amer. Library Ass., 2009.

30. Matthews J. Strategic Planning and Management for Library Managers, 168 p., Libraries Unlimited, 2005.

31.Sager D.J. Managing the Public Library (Professional Librarian Series), 322 p., G.K. Hall, 1989

32. Smith G.S. Managerial Accounting for Libraries \& Other Not-For-Profit Organizations, 204 p., Amer. Library Ass., 2002.

33. Martin S. Managing Without Managers: Alternative Work Arrangements in Public Organizations, 194 p., SAGE Pub., 1983.

34. Smith A.S., Lehrer M.D. Legacies for Libraries: A Practical Guide to Planned Giving, 138 p., Amer. Library Ass., 2000.

35.Commway L.S., Powell R.R. Basic Research Methods for Librarians (Library and Information Science Text Series), 370 p., Libraries Unlimited, 2010 .

36. Trosow S.E., Nolsen K.E. Constraining Public Libraries: The World Trade Organization's General Agreement on Trade in Services, 272 p., Scarecrow Press, 2006.

37.Burke J.J. Neal-Schuman Library Technology Companion, Third Edition, 279 p., Neal-Schuman Publishers, 2009.

38.Bell S.S. Librarian's Guide to Online Searching, 308 p., Libraries Unlimited, 2009 .

39. Sager D.J. Small Libraries: Organization and Operation, Upstart Books, 2000. 
40.Buck R.A., Gilmore J.A. Museum Registration Methods 5th Edition, 516 p., Amer. Ass. of Museums, 2010.

41. Yakel E. Starting an Archives, 112 p., Scarecrow Press, 1994.

42.Millar L.A. Archives: Principles and Practices, 256 p., Neal-Schuman Publishers, 2010.

43.O'Tolle J.M., Cox R.J. Understanding Archives \& Manuscripts (Archival Fundamentals Series), 237 p., Society of Amer. Archivists, 2006.

44.Roe K. Arranging \& Describing Archives \& Manuscripts,180 p., Society of Amer. Archivists, 2005.

45.Serrell B. Exhibit Labels: An Interpretive Approach, 270 p., AltaMira Press, 1996.

46. Giesecke J.R. The Dynamic Library Organizations in a Changing Environment, 126 p., Routledge, 1995.

47.Rikowski R. Globalization, Information and Libraries: The Implications of the World Trade Organization's GATS and TRIPS Agreements, 400 p., Neal-Schuman Publishers, 2005.

48.Preer J. Library Ethics, 272 p., Libraries Unlimited, 2008.

49. Bray I. The Volunteers' Guide to Fundraising: Raise Money for Your School, Team, Library or Community Group, 432 p., NOLO, 2011.

50.Hock R. The Extreme Searcher's Internet Handbook: A Guide for the Serious Searcher, 360 p., Information Today Inc., 2010.

51.Mann T. The Oxford Guide to Library Research, 320 p., Oxford University Press, 2005.

52.Howard N. The Book: The Life Story of a Technology, 192 p., Johns Hopkins University Press, 2009.

53.Нотченко А.В., Градов О.В. Методы лазерного баркодинга в идентификации и расшифровке нейрофизиологической информации. VIII Российско-Баварская конференция по биомедицинской инженерии (секция "Микро и нанотехнологии в биомедицинской инженерии"), сс. 175-180, СПб., 2012. 